\title{
TRABAJO Y FAMILIA Hacia una cultura familiar amigable en el contexto latinoamericano
}

\author{
Patricia Debeljuh, Ph.D. \\ PROFESORA INVESTIGADORA DE ÉTICA EMPRESARIAL \\ UNIVERSIDAD ARGENTINA DE LA EMPRESA \\ pdebeljuh@uade.edu.ar \\ Kety Jáuregui, Ph.D. \\ PROFESORA DE ADMINISTRACIÓN \\ DiReCTORA DEL PROGRAMA DOCTORAL ESADE-ESAN \\ kjauregui@esan.edu.pe
}

\section{Resumen}

Equilibrar el trabajo y la familia es la lucha diaria de toda persona que debe satisfacer las demandas, a menudo contrapuestas, de ambas esferas de su vida. La ausencia de soluciones eficaces a este problema no sólo afecta a la familia, sino también a las empresas a través del ausentismo laboral, la baja productividad, el incremento del estrés, entre otros efectos. Las condiciones de trabajo del futuro tendrán que resolver las tensiones entre los viejos paradigmas y las nuevas realidades y crear una cultura que refleje las características de la fuerza laboral actual. En la medida en que las compañías reconozcan y respondan a las necesidades familiares de sus empleados, desarrollarán estrategias para armonizar el trabajo y la vida familiar. El presente estudio pasa revista a esas estrategias y su implementación gradual, cuya última etapa es la creación de una cultura corporativa familiar amigable, único ámbito en el cual los empleados pueden lograr un equilibrio laboral-personal.

Equilibrar el trabajo y la familia, el ám-

— bito laboral y el ámbito doméstico, es la lucha diaria de toda persona que debe satisfacer las demandas, a menudo contrapuestas, de ambas esferas de su vida.

El mercado de trabajo ha sufrido grandes transformaciones en las últimas décadas. La tradicional división de tareas, por la cual los hombres salían a trabajar mientras las mujeres se dedicaban al cuidado del hogar, ha dado paso a un nuevo paradigma. Actualmente, unos y otras se plantean una carrera profesional compatible con las exigencias de la vida familiar, muchas veces presionados por necesidades económicas. De ahí que surjan conflictos y dilemas cuando se busca conci- 
liar las demandas de una profesión con las necesidades de una familia. En muchos casos, las presiones pueden llevar al agotamiento físico o nervioso o a aproximarse peligrosamente a éste.

Si bien algunas personas logran un satisfactorio equilibrio entre trabajo y familia por sus propios medios, la mayoría de ellas necesita el apoyo explícito de sus empresas para intentar alcanzarlo. Las empresas más modernas han aceptado el desafío y como un modo concreto de asumirlo implementan «políticas familiares amigables» (PFA).

Frente a esta problemática, el objetivo del presente artículo es mostrar, desde la perspectiva global y teniendo en cuenta el contexto de las empresas, cómo se puede llegar a adquirir una cultura familiar amigable en países latinoamericanos, donde el marco de la difícil situación económica agrega nuevos condicionamientos a la complejidad del tema.

En primer término se describen algunos aspectos del contexto latinoamericano y se resumen algunas concepciones sobre el trabajo y su significado. A continuación se analiza el papel de los distintos agentes involucrados en el conflicto familia-trabajo y se pasa revista a diversas alternativas para implementar una PFA. Finalmente, se muestra cómo puede configurarse una cultura corporativa, familiar y amigable, todo lo cual se recoge después en conclusiones y recomendaciones.

\section{El contexto latinoamericano}

En un mundo donde pareciera que los valores están en crisis, la familia no ha sido ajena a las profundas transformaciones que se han dado en las últimas décadas; la principal, sin duda, el ingreso masivo de las mujeres en el mercado de trabajo. Diversos factores han estado en el origen de este proceso: por un lado, la crisis económica mundial, muy intensa sobre todo en Latinoamérica, que obligó a las familias a la búsqueda de fuentes complementarias de ingresos; y por otro lado, el fenómeno de liberalización de la mujer en todos los aspectos, su acceso a la educación superior y su búsqueda de reconocimiento profesional.

Estos hechos han causado diversos impactos sobre la familia. Desde la disminución del índice de natalidad y el número de hijos por pareja, hasta el aumento de los divorcios y separaciones, pasando por el retraso de la maternidad -la mujer espera hasta la treintena para embarazarse-, el bajo rendimiento escolar de los niños y adolescentes, etc.

En cuanto al aspecto netamente económico, veamos algunas cifras. Según fuentes oficiales ${ }^{1}$, en el año 2002 el 45,3\% de los hogares argentinos se sostenían con dos ingresos, mientras en el Perú este porcentaje era similar: $43 \%$. Además, en ambos países se pudo constatar que hay muchas familias cuyo jefe de hogar es mujer y la única que aporta ingresos al núcleo familiar.

Para agravar la situación, el desempleo y subempleo ocasionan que la jornada laboral sea muy extensa. En pocas empresas se trabaja ocho horas, generalmente hombres y mujeres permanecen diez y hasta doce horas en sus centros de trabajo; en consecuencia, ni a los padres ni a

1. Datos tomados del INDEC de Argentina y del INEI del Perú. 
las madres les queda mucho tiempo para dedicarlo al hogar o a los hijos.

Más aún, no hay que olvidar que en épocas de altos índices de desempleo, los trabajadores no se atreven a plantear sus conflictos personales por temor a perder sus empleos, ante la gran oferta de mano de obra existente. En este contexto es difícil que las empresas, instituciones y gobiernos en Latinoamérica apoyen políticas de conciliación de trabajo y familia.

Cabe destacar que este problema no sólo se presenta en empresas de capitales nacionales, sino también en compañías multinacionales que están operando en países latinoamericanos. Por ejemplo, en Estados Unidos son muchas las empresas líderes por su política familiar amigable; sin embargo, fuera de sus fronteras estas mismas empresas operan bajo otros parámetros. Quizá porque consideran que el verdadero responsable de velar por los intereses de los trabajadores son los Estados, o tal vez porque creen que la realidad social o la idiosincrasia local las exime de asomarse a estos problemas.

Debe señalarse también el papel que juega en este tema una creencia muy arraigada en nuestras sociedades acerca de que cada quien debe resolver sus problemas personales, aunque ello signifique que algún miembro de la pareja -generalmente la mujer-deba renunciar al trabajo para dedicarse a la atención de los hijos.

Distinta es la situación en Europa, donde se está avanzando mucho en este tema debido a los bajos índices de natalidad y al surgimiento de problemas sociales. Chinchilla, Poelmans y León (2003) encuentran que en España algunas consecuencias del conflicto entre «trabajo y familia» son: estrés, divorcios, bajos índices de natalidad, padres que pasan muy poco tiempo con sus hijos y deben dejar la educación de éstos en manos de los abuelos o de las guarderías.

En cuanto a las llamadas estrategias de adaptación familiares (Moen y Yan Yu, 1999), que indican cómo actúa cada familia para conciliar trabajo y familia, la realidad latina difiere de la norteamericana o europea, principalmente porque aquí se cuenta con el apoyo de la familia extendida. Con frecuencia el cuidado de los niños pequeños o de los enfermos queda en manos de los abuelos u otros parientes cercanos. Otra diferencia proviene del relativo bajo costo de la mano de obra dedicada a tareas domésticas en nuestros países, por lo que también es frecuente que los niños queden bajo el cuidado de empleadas del hogar.

Estos condicionamientos de la realidad actual, lejos de desalentarnos en la tarea de conciliar el trabajo y la familia, constituyen un punto de partida adecuado para analizar cómo puede fomentarse una cultura familiar amigable dentro de un contexto que a priori no la favorece y aun cuestiona su relevancia.

\section{Viejas creencias y nuevas realidades sobre el trabajo}

Cuando las empresas se plantean asumir la responsabilidad de ofrecer a sus empleados opciones viables para equilibrar las exigencias del trabajo con las de su familia, a menudo deben enfrentarse a estereotipos cargados de prejuicios y temores. Por ejemplo, en muchas empresas el éxito profesional se mide sobre la base del tiempo que uno le dedica al trabajo o a 
reuniones informales relacionadas con el trabajo fuera del horario laboral.

En este sentido, Apgar (2000) menciona algunas ideas muy arraigadas: los problemas personales deben quedarse en casa; si se les facilita a los empleados la solución de sus problemas, terminarán reclamando cada vez nuevas exigencias; igualdad significa idéntico, es decir, «la misma solución para todos»; esos beneficios sólo pueden satisfacer a los trabajadores y mantenerlos contentos, pero no hacerlos más productivos.

Sin embargo, Litchfield, Swanberg y Sigworth (2004) sostienen algunos beneficios que obtienen las empresas al implementar PFA: mayor lealtad y grado de identificación con la empresa; disminución de los índices de absentismo; menor estrés en los trabajadores; incremento de la productividad debido a mayor concentración; reducción de costos fijos; mayor retención de empleados competentes, con mejores resultados en los costos de capacitación de los mismos; mayores niveles de satisfacción laboral; mayor captación de trabajadores altamente calificados; todo ello porque incluso en situaciones de aumento de las horas de trabajo los empleados se sienten a gusto con el equilibrio logrado.

Si bien estos aspectos pueden ser ilustrativos y motivadores para las empresas, es preciso dar un paso más para no plantear estas ventajas con un tinte utilitarista o de mera conveniencia que va en detrimento de la verdadera naturaleza de la cuestión.

Familia y trabajo no son ámbitos que compiten entre sí, sino más bien se complementan y a la vez se contraponen, por- que en ellos se centra el desarrollo personal (Apgar, 2000). En efecto, desde lo más íntimo de su ser, cada persona es llamada a alcanzar su plena satisfacción tanto en el mundo laboral como en el familiar. Ambas realidades están íntimamente relacionadas. De hecho, el trabajo adquiere significado en la medida en que es el medio para el sustento de las personas que uno quiere $y$, por lo tanto, se trabaja para la propia familia, con el objetivo de fortalecerla y desarrollarla.

La familia es el primer ámbito irrenunciable donde la persona desarrolla todas sus potencialidades y ejerce una dimensión propia: darse a sí mismo. No obstante, por lo general se considera que esta cuestión atañe sólo a las mujeres, porque se la asocia exclusivamente con la maternidad. Pero no es así, también el hombre ha de dedicar tiempo y entregar sus mejores energías dentro del hogar, porque no puede renunciar a una de las principales facetas de su personalidad: ser esposo, padre, hijo o abuelo.

Por su parte, el trabajo es otro ámbito de perfeccionamiento personal en el cual el individuo despliega otras capacidades y las pone al servicio de los demás. El trabajo tiene que llevar a un verdadero desarrollo personal. En este sentido, Melendo (1994) afirma que un trabajo no puede ser personalmente enriquecedor cuando se realiza de manera tal que impide al ser humano -por cantidad o calidad- ejercer las funciones más nobles de su personalidad en el desempeño de su labor o fuera de ella.

Por lo tanto, es preciso superar esta concepción de exclusivo incremento económico y darle al tema de la PFA un sentido más humanista. Al respecto, Melen- 
do (1994) sostiene «que la empresa debe considerarse como auténtica forja de humanidad, crisol de personalidades maduras y equilibradas, gracias al trabajo humanizado que ofrece a quienes colaboran con ella». En síntesis, la organización ha de supeditarse al desarrollo de las personas. Sólo desde esta perspectiva se entiende la responsabilidad que le cabe en esta tarea de perfeccionar al hombre y ayudarlo a que pueda desarrollarse en los dos ámbitos planteados: el trabajo y la familia.

\section{Tres pilares para fomentar el equilibrio entre trabajo y familia}

Sin intentar dar una receta simplista a un problema muy controvertido, puede afirmarse que la respuesta sobre cómo equilibrar las demandas del trabajo y de la familia debe provenir de tres frentes: el propio individuo o trabajador, el Estado y la empresa. Es de fundamental importancia darse cuenta de que un equilibrio justo sólo se logrará con la intervención simultánea de estos tres pilares, pues cada uno por sí solo no podrá resolver el problema.

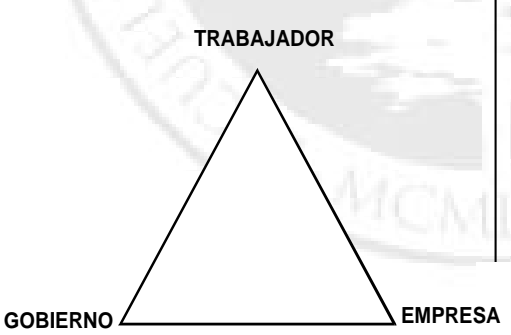

Los trabajadores deben implementar sus propias estrategias de adaptación familiar que les permitan armonizar su dedicación a la familia con las exigencias de su empleo. Por ejemplo, en el caso de un matrimonio será necesario conciliar las expectativas y perspectivas profesionales de cada cónyuge para definir los objetivos de la pareja.

El Estado debe brindar el respaldo institucional que necesita la familia, núcleo donde se cimentan los valores de la sociedad misma. Su responsabilidad en este tema lo obliga a velar por los intereses familiares y a diseñar un marco legal que favorezca el equilibrio familiar-laboral y cuyo alcance incluya a todos los sectores de la sociedad, sobre todo a los más desfavorecidos. La tarea del Estado se extiende también a promulgar leyes que faciliten a las empresas aplicar políticas familiares amigables y a brindar una sólida seguridad social que salvaguarde los derechos de la familia.

La empresa debe asegurar el bienestar de sus empleados y crear un ambiente laboral de apertura que los ayude a equilibrar sus necesidades familiares con su desempeño profesional. El trabajo a medio tiempo, a distancia o compartido, así como una guardería in situ son alternativas que hacen de una empresa un lugar amigable para la familia. Pero más allá de las formas específicas de implementación, lo fundamental es que la empresa mantenga una política de trabajo que permita que cada uno pueda desarrollar sus tareas de acuerdo con sus necesidades particulares, sin ser desplazado u hostigado por estas mismas circunstancias.

\section{4. ¿Cómo implementar políticas familiares amigables?}

Gallinsky, Friedman y Hernández (1991) sostienen que las empresas pasan por tres 
etapas antes de llegar a implementar las PFA con éxito: desarrollo de una visión pragmática; desarrollo de una visión integradora y desarrollo de una cultura familiar amigable.

En la primera etapa, desarrollo de una visión pragmática, los directivos hacen frente a las críticas que el tema suscita e implementan soluciones parciales a fin de remediar problemas concretos de algunos trabajadores. En esta etapa es común la implantación de un servicio extraordinario de guardería, por ejemplo. Sin embargo, con frecuencia se descubre que los empleados beneficiados por alguna medida de este tipo deben enfrentar nuevos problemas: la hostilidad que en sus compañeros provoca este supuesto «trato de favor». Al margen del estrés que esta situación acarrea, el no implementar las PFA de manera formal y para toda la organización origina malestar en el resto de empleados, que se sienten excluidos porque no pueden beneficiarse del mismo modo. Dado que los esfuerzos generalmente están concentrados en el cuidado de los niños, estas medidas se consideran como un beneficio sólo para las madres $\mathrm{y}$, por ende, discriminatorias a favor de ellas. Forzados a aceptar que es un beneficio para padres y madres, los trabajadores varones insisten en que sólo beneficia a unos pocos padres con hijos pequeños. El problema, en consecuencia, reclama otro tipo de solución que pueda brindar opciones para todos.

En la segunda etapa se pasa de una concepción fragmentaria a una visión que interrelaciona los esfuerzos, esto es, se desarrolla una visión integradora que abarca un espectro más amplio de cuestiones y considera a las distintas PFA implementadas como parte de una estrate- gia global. En esta etapa hay que tener en cuenta lo siguiente: i) el compromiso gerencial, ya que gran parte del éxito de las PFA dependerá de que su creación sea impulsada por la alta gerencia -y mucho mejor si se crea un área o cargo específico relacionado con los temas de familiatrabajo dentro de la organización-; ii) el involucramiento del área de Recursos Humanos, que promueve las PFA; iii) la integración de políticas y programas, fundamental para que los trabajadores puedan realmente hacer uso de ellos; por ejemplo, si la empresa ofrece un servicio de guarderías, de poco les servirá a los trabajadores si no se les da facilidades para ausentarse del trabajo y entrevistarse con profesionales que ofrecen esos servicios; y iv) la flexibilidad en los puestos de trabajo, que comprende el trabajo a medio tiempo, el horario flexible, permisos y días libres para propósitos específicos, y otras variantes de absentismo diseñadas a la medida de las necesidades de cada trabajador.

Finalmente, en la tercera etapa se consolida una cultura familiar amigable sobre la base de valores corporativos que asumen una verdadera cultura familiar amigable. Las PFA pueden ser consideradas como un medio para alcanzar entre todos una auténtica cultura que impregne a la organización en su conjunto. Puede decirse entonces que las políticas y la cultura corporativa están relacionadas hasta tal punto que ésta puede mejorar o anular el desarrollo y la efectividad de aquéllas (Starrels, 1992).

De esta manera, se comprende que las distintas PFA ofrecidas sólo pueden ser viables dentro del marco de una cultura corporativa que las acoja. En consecuencia, algunas empresas pioneras están in- 
tentando transformar sus culturas para hacerlas más familiarmente amigables, comenzando por aceptar públicamente que sus empleados tienen vidas personales además de laborales. Si hay éxito, las PFA pueden llegar a constituir una verdadera cultura dentro de la organización, entendida ésta como «un conjunto de creencias -inventadas, descubiertas o desarrolladas por un grupo a medida que aprende a afrontar sus problemas de adaptación externa y de integración internaque ha funcionado suficientemente bien para ser juzgada válida y, consiguientemente, para ser enseñada a los nuevos miembros como el modo correcto de percibir, pensar y sentir sobre estos problemas» (Schein, 1985: 9).

De esta definición se desprende que la propia cultura contiene la respuesta a las dos grandes categorías de problemas que enfrenta una organización y que pueden aplicarse también a la tarea de conciliar las exigencias profesionales y familiares. Se trata de la adaptación al entorno y la integración interna.

En el ámbito de la adaptación al entorno, cada organización ha de desarrollar una misión, definir unos objetivos y formular e implementar una estrategia. Por consiguiente, la empresa traduce su compromiso de equilibrar trabajo y familia desde la misión y objetivos que persigue hasta el modo de organizar las tareas. Por una parte, estará transmitiendo un mensaje coherente a toda la organización y, por otra, implementará los sistemas necesarios para que las PFA se hagan realidad.

Lo mismo puede aplicarse a la integración interna. Rodríguez Porras (1994) sostiene que cada organización ha de desarrollar un lenguaje y unas categorías conceptuales comunes, unos criterios sobre la clase de personas que quiere incorporar como miembros, sobre el poder y el estatus, sobre el tono de las relaciones interpersonales -el grado de apertura de intimidad-, sobre lo que está bien y lo que está mal, sobre el modo de premiar y de castigar; para así afianzar su propia cultura.

Finalmente, es importante señalar que a estas tres etapas, íntimamente relacionadas y progresivamente concatenadas, Bankert y Litchfield (1997) agregan una cuarta: comunicación a las partes interesadas. En efecto, una vez que las PFA se han establecido como una cultura, es preciso transmitirlas no sólo dentro de la propia organización, sino también a todos los grupos de interés, tanto internos como externos a la empresa (tales como los accionistas, directivos, empleados, proveedores, clientes y comunidad en general).

\section{Hacia una cultura corporativa, familiar y amigable}

Para empezar, es preciso señalar que cualquier cultura corporativa que promueva acceso a las PFA debe premiar la innovación y, por ello, ser abierta y apoyar el cambio. En un estudio realizado por Bankert y Litchfield (1997), se muestra que las empresas estadounidenses más innovadoras y las que reciben más alta puntuación como familiarmente amigables son las que tienen una cultura que apoya la utilización de los beneficios ofrecidos. Por ejemplo, los trabajadores se sienten más contentos si se les facilita una lista de guarderías disponibles entre las cuales elegir, en lugar de una guardería en su lugar de trabajo, ya que ésta no beneficia a todos los empleados. 
Por el contrario, la adopción de políticas familiares amigables es ineficaz si se ve rodeada de una cultura corporativa hostil que imposibilita a los trabajadores acceder a los beneficios implementados. En este sentido, Pleck (1994) sostiene que una actitud cerrada por parte de los directivos impide a los padres utilizar los permisos ofrecidos y que, llegado el caso, son más proclives a aprovecharlos si no son llamados «permisos por paternidad».

En segundo lugar, la cultura corporativa que incluya PFA debe ser apoyada enérgicamente por los líderes de la organización. Las personas son muy sensibles y muchas veces descubren que la política y la práctica frecuentemente se oponen, así como también se contradicen el apoyo formal y el informal (Raabe, 1990). Como sostiene Starrels (1992), la mayor parte de las veces los directivos no apoyan estas políticas a pesar de haberlas implementado de un modo formal, o se dejan llevar por aquellas creencias tradicionales acerca de cómo se debe trabajar. En otras ocasiones, la implementación de políticas progresistas se ve perjudicada por actitudes negativas o una cultura corporativa que no promueve el cambio. En este contexto, Starrels (1992) recomienda la necesidad de capacitar a los directivos para que comprendan la vital importancia de mantener a los empleados satisfechos con su trabajo y libres de presiones ante las exigencias familiares. Se trata de que ellos mismos ayuden a sus empleados a resolver los posibles conflictos entre familia y trabajo y de que admitan que esta tarea es parte de sus responsabilidades.

En tercer lugar, siguiendo a Schein (1983), podemos proponer unas reflexio- nes en torno a lograr una cultura familiar amigable:

- Cuando las PFA llegan a conformar una verdadera cultura pasan a formar parte del credo corporativo, entendido éste como la misión que la empresa asume y por la cual quiere ser reconocida en su medio. Así, por ejemplo, Johnson \& Johnson ha incluido en su credo corporativo una referencia explícita a este tema, expresada así: «Debemos reconocer la forma de ayudar a nuestros empleados a cumplir con sus responsabilidades familiares».

- El directivo debe plantearse si es factible implementar formas alternativas de trabajo (entendido éste como una combinación de prácticas, entornos y puestos de trabajo no tradicionales), tales como oficina satélite, trabajo desde casa o trabajo compartido con otra persona.

- El directivo que dirige la organización tiene un papel clave en cuanto a ayudar a los empleados a equilibrar sus vidas profesionales y familiares. El ejemplo personal que él transmite, unido a sus indicaciones y objetivos, marca el nivel de la cultura que se desea implantar.

- Los sistemas para la asignación de premios y de estatus han de basarse en los resultados, independientemente de las horas que la persona dedique físicamente a las tareas. También se permitirán métodos no tradicionales de desarrollo en las carreras profesionales, incluso periodos de estancamiento a elección del propio trabajador que opte por no aceptar puestos de mayor exigencia en determinados momentos de su vida. 
- El líder será el primero en discutir abiertamente sus propios conflictos entre trabajo y familia, para incentivar a otros a seguir su ejemplo y tomar las medidas necesarias para resolverlos.

- El líder procurará transmitir un mensaje claro y homogéneo a todos los niveles de la empresa, de modo que las prácticas informales no contradigan la cultura oficial. Por ejemplo, evitará organizar reuniones a última hora o durante los fines de semana para no restarle tiempo de dedicación a la familia.

- La familia no debe ser considerada una desventaja o una carga cuando se trate de elegir o promover a una persona. Más aún, hay personas altamente calificadas que eligen trabajar en determinadas empresas conocidas por su política de apoyo a la familia.

- Los directivos deben apoyar abiertamente las PFA y hacer ellos mismos uso de ellas. Así, los trabajadores podrán comprobar que estas prerrogativas no pondrán en peligro su puesto de trabajo. En este sentido, Raabe (1990) menciona que la mayor parte de los empleados no aprovechan las PFA porque temen poner en riesgo su empleo o sus perspectivas de carrera dentro de la organización.

- Algunas empresas han redefinido el concepto de familia para incluir en ella a todas las personas que viven con el trabajador (significant others) o que, de alguna manera, dependen de él. Otras definen el tema bajo el epígrafe más amplio de trabajo-vida, lo que supone reconocer que en el transcurso de su vida el trabajador tendrá distin- tas personas a su cargo y, por lo tanto, tendrá que resolver diversos conflictos de trabajo y familia. Por ejemplo, en algún momento sólo le preocuparán sus hijos pequeños, más adelante deberá ocuparse también de sus padres ancianos.

Finalmente, es importante resaltar que para que la cultura de una organización sea valiosa hacen falta dos cualidades clave: la coherencia y la consistencia (Ribes Pons, 1997). La coherencia se expresa como la no contradicción entre las creencias y las pautas de comportamiento; en otras palabras, que las decisiones de un directivo no se opongan a los valores que encierra la cultura corporativa. La consistencia, por su parte, indica la intensidad con que los miembros de una organización comparten esos valores y hacen propia esa cultura. En la medida en que estas dos variables estén presentes y cada vez más afianzadas, la cultura familiar amigable será una realidad dentro de la organización y una meta compartida por todos.

\section{Conclusiones y recomendaciones}

El actual lugar de trabajo tiene que resolver las tensiones entre las viejas creencias y los prejuicios, por un lado, y las nuevas realidades, por otro lado. Se trata de crear una nueva cultura que refleje la diversidad de la fuerza laboral actual. En la medida en que el Estado y las empresas tomen conciencia de su responsabilidad en este tema y reconozcan las necesidades personales de sus ciudadanos y empleados, respectivamente, desarrollarán estrategias para armonizar el trabajo y la vida familiar. 
El marco actual de la economía latinoamericana no es el más favorable para implementar las PFA, pero tampoco puede ser un obstáculo para intentarlo. La empresa, aun cuando sufra los efectos de una compleja realidad socioeconómica, puede y debe encaminar sus esfuerzos hacia la configuración de esta cultura. Aunque en una primera instancia implemente medidas pequeñas, irá marcando una tendencia y configurando una cultura que realmente ayude a equilibrar los ámbitos familiar y laboral. De esta manera, las personas tomarán cada vez más conciencia de la importancia de conciliar trabajo y familia y ellas mismas podrán plantear alternativas, teniendo en cuenta la realidad actual y sus necesidades.

El punto de partida para lograr una acertada combinación entre la vida familiar y el ámbito laboral es que la empresa se comprometa con el desarrollo humano de quienes trabajan en ella. De ahí la necesidad de incluir los asuntos de trabajo-familia como parte fundamental de las políticas de recursos humanos de la organización y comunicar esta importancia claramente a los empleados. La comunicación es clave para la configuración de una cultura, unida a la coherencia para respaldar con los hechos lo que se ha comunicado.

Por otro lado, también es necesario escuchar las inquietudes de los empleados, ya que no hay mejor fuente de información que la que proviene de los mismos interesados, y no es útil aplicar una política que no resuelva los conflictos específicos planteados. En esta línea es importante conocer las circunstancias demográficas de los trabajadores, las necesidades de cada uno y sus exigencias familiares. A partir de este conocimiento se podrá estudiar con cada persona la mejor manera de afrontar los posibles conflictos.

Asimismo, la empresa debe diseñar adecuadamente el trabajo de sus empleados, desde objetivos claramente definidos hasta una cierta flexibilidad -así los trabajadores podrán tener control sobre dónde, cómo y cuándo realizar sus tareas, quizás un teletrabajo o un empleo a medio tiempo-. A partir de aquí se pueden establecer distintas políticas que ayuden a conciliar las demandas del trabajo y la familia. Sin embargo, su mera implementación no es suficiente; es preciso que estén enmarcadas dentro de una cultura familiar amigable y que lleguen a configurar un auténtico marco de pensar y actuar dentro de cada organización. De este modo, los empleados no sólo se sentirán libres de utilizar las soluciones que la empresa les ofrece, sino también tendrán la confianza y el estímulo para participar y aportar otras alternativas adaptadas a sus necesidades particulares.

Por último, es importante diseñar un plan de formación dirigido a los ejecutivos para desterrar viejos estereotipos, implementar en su lugar nuevas directivas y transmitir la convicción de que la empresa adoptará una clara política de apoyo a la familia. 


\section{Referencias bibliográficas}

APGAR, M. 1998. The alternative workplace: Changing how and where people work. Harvard Business Review. Boston, vol. 76, n. ${ }^{\circ}$, págs. 121-136.

BANKERT, E. y LITCHFIELD. 1997. Business week's work and family corporate ranking: an analysis of the data. Boston: Boston College, Carroll School of Management.

CHINCHILLA, POELMANS y LEÓN. 2003. Políticas de conciliación trabajo-familia en 150 empresas españolas. Barcelona: IESE, Universidad de Navarra.

DE MARCO, G. M. 1995. La familia en la Argentina de fines de siglo: aporte geodemográfico. Buenos Aires: Consejo Nacional de Investigaciones Científicas y Técnicas.

ELDER, G. H. Jr.; CONGER, R.; FOSTER, M. y ARDELT, M. 1992. Families under economic pressure. Journal of Family Issues. Vol. 13, n. ${ }^{\circ} 1$, págs. 5-37.

FAMILIES AND WORK INSTITUTE. 1993. An Evaluation of Johnson and Johnson's Balancing Work and Family Program. New York: Families and Work Institute.

FERBER, Marianne A.; O'FARRELL, Brigid y LA RUE, Allen. 1991. Work \& family: Policies for a changing work force. Washington D.C.: National Academic Press.

FRIEDMAN, S.; CHRISTENSEN, P. y DEGROOT, J. 1998. Work and life: The end of the zero-sum game. Harvard Business Review. Boston, vol. 76, n. ${ }^{\circ}$ 6, págs. 119-129.
GALLINSKY, E.; FRIEDMAN, D. y HERNÁNDEZ, C. 1991. The corporate reference guide to work family programs. New York: Families and Work Institute.

LITCHFIELD, SWANBERG y SIGWORTH. 2004. Increasing the visibility of the invisible workforce: Model programs and policies for hourly and lower wage employees. Boston: Boston College Center for Work \& Family, Carroll School of Management.

LLANO, A. y LLANO, C. 1999. Paradojas de la ética empresarial. Empresa y Humanismo. Pamplona, Universidad de Navarra, vol 1, n. $^{\circ} 1$, págs. 69-89.

MELENDO, T. 1994. El trabajo, medio de desarrollo personal. En: Melé, D. Ética, trabajo y empleo. Pamplona: EUNSA.

MOEN, P. y YAN YU. 1999. Effective work/ life strategies: Working couples, gender and life quality. New York: Cornell University. Working Paper Series, Bonfenbrenner Life Course Center.

NACIONES UNIDAS. 2000. La familia $y$ el mundo del trabajo: cuatro estudios nacionales seleccionados de políticas que tienen en cuenta a la familia. Naciones Unidas: Nueva York.

PLECK, J. 1994. Family supportive employer policies and men: A perspective. Center for Research of Women, Working Paper Series $n^{\circ} 274$.

RAABE, P. H. 1990. The organizational effects of workplace family policies: Past 
weaknesses and recent progress toward improved research. Journal of Family Studies. N. ${ }^{\circ} 11$, págs. 477-491.

RIBES PONS, A. 1997. La cultura en la empresa: cómo actuar en ella y desde ella. Harvard Deusto Business Review. Julioagosto.

RODRÍGUEZ PORRAS, J. M. 1994. El factor humano en la empresa. Bilbao: Deusto.
SCHEIN, E. 1983. The role of the founder in creating organizational culture. Organizational dynamics. New York, American Management Association, vol 12, n. ${ }^{\circ}$, págs. 13-28.

-1985. Organizational culture and leadership. San Francisco: Jossey-Bass.

STARRELS, M. 1992. The evolution of workplace family policy research. Journal of Family Issues. N. ${ }^{\circ} 13$, págs. 259-278. 